Vietnam Journal of Mechanics, VAST, Vol.38, No. 4 (2016), pp. 239 - 248

DOI:10.15625/0866-7136/6753

\title{
EQUIVALENT-INCLUSION APPROACH FOR THE CONDUCTIVITY OF ISOTROPIC MATRIX COMPOSITES WITH ANISOTROPIC INCLUSIONS
}

\author{
Do Quoc Hoang ${ }^{1,2, *}$, Pham Duc Chinh ${ }^{2,3}$, Tran Anh Binh ${ }^{1,2}$ \\ ${ }^{1}$ National University of Civil Engineering, Hanoi, Vietnam \\ ${ }^{2}$ Graduate University of Science and Technology, VAST, Hanoi, Vietnam \\ ${ }^{3}$ Institute of Mechanics, VAST, Hanoi, Vietnam \\ *E-mail: hoangdq@nuce.edu.vn \\ Received August 15, 2015
}

\begin{abstract}
Many effective medium approximations for effective conductivity are elaborated for matrix composites made from isotropic continuous matrix and isotropic inclusions associated with simple shapes such as circles or spheres, ... In this paper, we focus specially on the effective conductivity of the isotropic composites containing the disorderly oriented anisotropic inclusions. We aim to replace those inhomogeneities by simple equivalent circular (spherical) isotropic inclusions with modified conductivities. Available simple approximations for the equivalent circular (spherical)-inclusion media then can be used to estimate the effective conductivity of the original composite. The equivalentinclusion approach agrees well with numerical extended finite elements results.
\end{abstract}

Keywords: Isotropic multicomponent material, disorderly oriented anisotropic inclusions, effective conductivity, matrix composite, equivalent inclusion.

\section{INTRODUCTION}

Theoretical determination of effective properties of multicomponent materials generally is difficult because of their complex microstructure and the random distribution of inclusions. The most rigorous approach is to to construct upper and lower bounds on the possible values of the effective properties, [1-3]. The bounds containing the properties and volume fractions of the component materials are not very useful in the case of high contrast of matrix-inclusion properties. The numerical methods [4-9], such as finite element one, fast Fourier transformation may give better results. However, they require much computer resources and computational time when the microstructure is complex and the inclusions are close to each other.

Engineers would prefer more simple effective medium approximations developed over years from the most simple arithmetic and harmonic volume-weighted averages to

(C) 2016 Vietnam Academy of Science and Technology 
more advanced self-consistent, differential, Mori-Tanaka approximation, correlation approximation... [10-17], which often account just for the shapes of the inhomogeneities and the matrix-inclusion interactions. Many approximations are actually applicable for only the composites with isotropic inclusions of simple shapes. The main objective here is to construct a novel approach for macroscopically isotropic composites containing the disorderly oriented anisotropic inclusions, which substitutes those inhomogeneities by circular (spherical) isotropic inclusions with equivalent conductivity. Above approximations then can be used to estimate the conductivity of original media. The layout of this paper is as follows.

First, we present the details of the proposed approach in section 2 . In section 3 , the effective medium approximation for effective conductivity of macroscopically isotropic composites containing the disorderly oriented anisotropic inclusions are compared with extended finite element results for some composites with complex microstructure, including that associated with random geometry. The conclusion completes the paper.

\section{THE EQUIVALENT-INCLUSION APPROACH}

Let us consider a d-dimensional macroscopically isotropic medium associated with anisotropic particles of certain shapes. The effective conductivity of a dilute suspension of those randomly-oriented particles having principal conductivities $c_{I 1}, c_{I 2}, \ldots, c_{I d}$ and volume proportion $v_{I}$ in a matrix of conductivity $c_{M}$ has the form

$$
c^{e f f}=c_{M}\left[1+v_{I} D\left(c_{I 1}, \ldots, c_{I d}, c_{M}\right)+O\left(v_{I}^{2}\right)\right] .
$$

In the meantime, the dilute suspension of circular (spherical) particles having conductivity $\bar{c}_{I}$ and volume proportion $v_{I}$ in the matrix of the same conductivity $c_{M}$ has the particular expression

$$
c^{e f f}=c_{M}\left[1+v_{I} \frac{2\left(\bar{c}_{I}-c_{M}\right)}{\left[\bar{c}_{I}+(d-1) c_{M}\right]}\right] .
$$

Equalizing (1) and (2), one finds

$$
\bar{c}_{I}=c_{M} \frac{D\left(c_{I 1}, \ldots, c_{I d}, c_{M}\right)(d-1)+2}{2-D\left(c_{I 1}, \ldots, c_{I d}, c_{M}\right)} .
$$

In the special case the anisotropic inclusions have the circular (spherical) shape, (1) has the particular expression [18]

$$
c^{e f f}=c_{M}\left[1+v_{I} \sum_{i=1}^{d}\left(\frac{c_{I i}-c_{M}}{c_{I i}+(d-1) c_{M}}\right)\right], \quad v_{I} \ll 1 .
$$

In that case

$$
D\left(c_{I 1}, \ldots, c_{I d}, c_{M}\right)=\sum_{i=1}^{d}\left(\frac{c_{I i}-c_{M}}{c_{I i}+(d-1) c_{M}}\right) .
$$

$\bar{c}_{I}$ is referred to as the conductivity of the equivalent circular (spherical) inclusion for the original inclusions of conductivity $c_{I}$. 
The simplest approximation for the conductivity of matrix circular (spherical)inclusion composites is the Maxwell one $[17,19]$, which will be used in applications with $\bar{c}_{I}$ taking the place of $c_{I}$.

\section{NUMERICAL EXAMPLES FOR EFFECTIVE CONDUCTIVITY}

\subsection{Basic of XFEM/Level-set method}

In this section, we use the Maxwell Approximation (MA) and eXtended Finite Elements method (XFEM) to estimate the conductivity of the isotropic composite containing the disorderly oriented anisotropic inclusions. Introducing equivalent inclusion with conductivity $\bar{c}_{I}$ defined in (3) instead of $c_{I}$ in the polarization formula [16], which is similar to Maxwell one, one finds

$$
c^{e f f}=P\left(c_{M}\right),
$$

where

$$
P\left(c_{0}\right)=\left[\frac{v_{M}}{c_{M}+c_{0}}+\frac{v_{I}}{2}\left(\frac{1}{c_{I 1}+c_{0}}+\frac{1}{c_{I 2}+c_{0}}\right)\right]^{-1}-c_{0} .
$$

For numerical computations, the extended finite elements method [20-24] will be used. In this framework, the level-set function is involved to describe the interface of inclusions. The essence of this method is to enrich the finite element approximation with additional functions to model interfaces or singularities independently of the background mesh. So the XFEM does not require a mesh conforming with all internal surfaces of inclusions as the finite element mesh.

Consider a domain $\Omega \subset \mathbf{R}^{D}$ with boundary $\partial \Omega$ ( $D$ being the dimension of the domain) which is partitioned into finite elements, and $\mathcal{S} \subset \mathbf{N}^{*}\left(\mathbf{N}^{*}\right.$ being the set of positive natural numbers) be a set of $N$ nodes indices in the mesh. The XFEM temperature field approximation can be expressed by

$$
T^{h}(\mathbf{x})=\sum_{i \in S} N_{i}(\mathbf{x}) T_{i}+\sum_{j \in \mathcal{S}^{e}} N_{j}^{*}(\mathbf{x}) \psi(\mathbf{x}) a_{j} .
$$

In the above equation, $T_{i}$ and $a_{j}$ are nodal unknowns, $N_{i}(\mathbf{x})$ and $N_{j}^{*}(\mathbf{x})$ are finite element shape functions, not necessarily the same. The nodal set $\mathcal{S}^{e}$ is defined as:

$$
\mathcal{S}^{e}=\left\{j \mid j \in \mathcal{S}, \omega_{j} \cap \Gamma \neq \varnothing\right\},
$$

where $\Gamma$ is an interface, that does not necessarily coincide with the mesh. $\omega_{i}=\operatorname{supp}\left(n_{i}\right)$ is the support of the nodal shape function $N_{i}$, which consists of the union of all elements connected to the node $n_{i}$. The function $\psi(\mathbf{x})$ is an enrichment function with the desirable discontinuous properties. In this work, the enrichment function $\psi(\mathbf{x})$ can be chosen in the form proposed by Moës et al. [20]

$$
\psi(\mathbf{x})=\sum_{i}\left|\phi_{i}\right| N_{i}(\mathbf{x})-\left|\sum_{i} \phi_{i} N_{i}(\mathbf{x})\right| .
$$

where $\phi(\mathbf{x})$ is level-set function $[20,23]$. 
The field equations of thermo-static problem are given by

$$
\begin{aligned}
& \nabla \cdot \mathbf{q}(\mathbf{x})-r(\mathbf{x})=0 \text { in } \Omega, \\
& \mathbf{q}(\mathbf{x})=-\mathbf{C}(\mathbf{x}) \nabla T(\mathbf{x}) .
\end{aligned}
$$

Above, $\mathbf{q}(\mathbf{x})$ denotes the heat flux, $r(\mathbf{x})$ is a heat source term and $c(\mathbf{x})$ the conductivity tensor. The periodic boundary conditions are: q.n is antiperiodic on $\partial \Omega$, and $T$ is periodic on $\partial \Omega$. $(\mathbf{C})$ is the conductivity matrix. More precisely, $\mathbf{C}(\mathbf{x})=\mathbf{C}_{\mathbf{I}}$ for anisotropic inclusions is defined as

$$
\mathbf{C}_{\mathbf{I}}=-\mathbf{R}^{\mathrm{T}} \mathbf{C}^{*} \mathbf{R}
$$

where $\mathbf{C}^{*}$ is conductivity matrix in local coordinate system $(1,2)$, which reads as

$$
\mathbf{C}_{\mathbf{I}}^{*}=\left[\begin{array}{cc}
c_{I 1} & 0 \\
0 & c_{I 2}
\end{array}\right]
$$

and $\mathbf{R}$ is rotation matrix expressed by

$$
\mathbf{R}=\left[\begin{array}{cc}
\cos \theta & -\sin \theta \\
\sin \theta & \cos \theta
\end{array}\right]
$$

The weak form associated with Eqs. (11)-(12) is given by finding $T \in \mathcal{D}=\{T \mid T=$ $\bar{T}$ on $\partial \Omega_{T}, T$ in $\left.H^{1}(\Omega)\right\}$ such that

$$
\int_{\Omega} \mathbf{q} \nabla(\delta T) d \Omega+\int_{\Omega} r \delta T d \Omega=0,
$$

where $H_{0}^{1}(\Omega)=\left\{\delta T \mid \delta T \in H^{1}(\Omega), \delta T=0\right.$ on $\left.\partial \Omega_{T}\right\}$.

By substituting the temperature field defined in (8) into the weak form (16) we obtain the discrete system of linear ordinary equations

$$
\mathbf{K d}=\mathbf{Q}, \quad \mathbf{d}=\left\{\begin{array}{ll}
\mathbf{T} & \mathbf{a}
\end{array}\right\}^{T},
$$

where $\mathbf{d}$ are nodal unknowns and $\mathbf{K}$ and $\mathbf{Q}$ are the global stiffness matrix and external flux, respectively. More precisely, the matrix $\mathbf{K}$ and vector $\mathbf{Q}$ are defined by

$$
\mathbf{K}=\int_{\Omega} \mathbf{B}^{T} \mathbf{C}(\mathbf{x}) \mathbf{B} d \Omega, \mathbf{Q}=\int_{\Omega} \mathbf{N}^{T} r d \Omega,
$$

where $\mathbf{B}$ and $\mathbf{N}$ are the matrices of shape function derivatives and shape functions associated with the approximation scheme (8).

The periodic boundary condition is finally introduced to (17) by mean of multiplicator Lagrange.

\subsection{Numerical example}

As a numerical example we consider the two-component 2D square-periodic suspension of anisotropic inclusions having conductivity $c_{I 1}$ and $c_{I 2}$ in a matrix of conductivity $c_{M}$ (Fig. 1(a)) (the principal axis is shown in the figure, secondary axis is perpendicular to the principal one), which have macroscopically isotropic conductivity $c^{\text {eff }}$. For the next model, we consider the hexagonal-periodic suspension with position of inclusions being located same as in Fig. 1(b). The local coordinate system $(1,2)$ makes an angle $\theta$ with 
the global coordinate system $(x, y)$ of the square cell. In the random model (Fig. 1(c)), the position of inclusions is generated with non-penetration algorithm; The radii of the inclusions vary from $0.035 \mathrm{~L}$ to $0.08 \mathrm{~L}, L$ being the dimension of the square cell. The equivalent configuration is that with circular (spherical) inclusions of the same centers and same volumes as the original one, but with different isotropic conductivity $\bar{c}_{I}$. The regular mesh

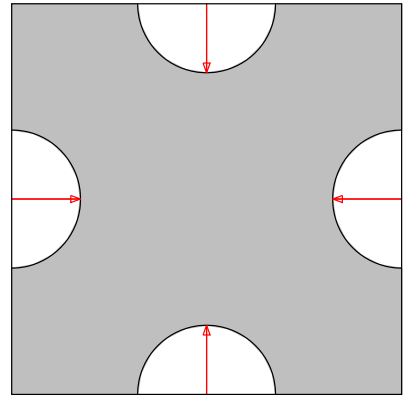

(a) The square cell

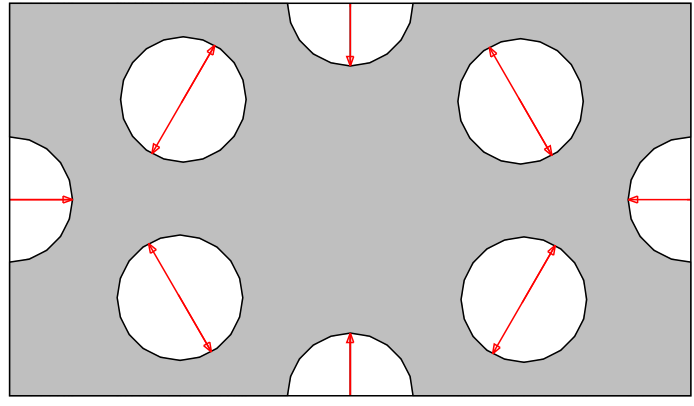

(b) The hexagonal cell

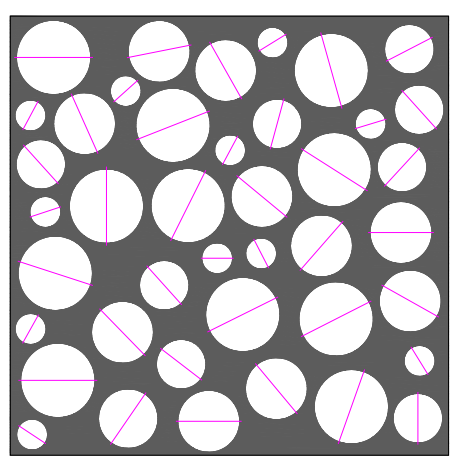

(c) The random cell

Fig. 1. The periodic cells of disorderly anisotropic inclusions having circular shape

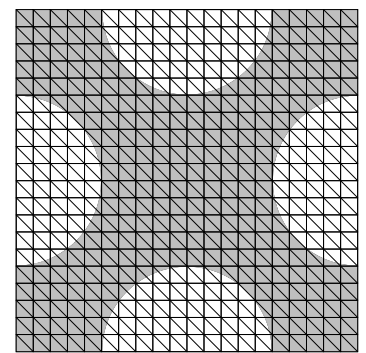

(a) The square cell

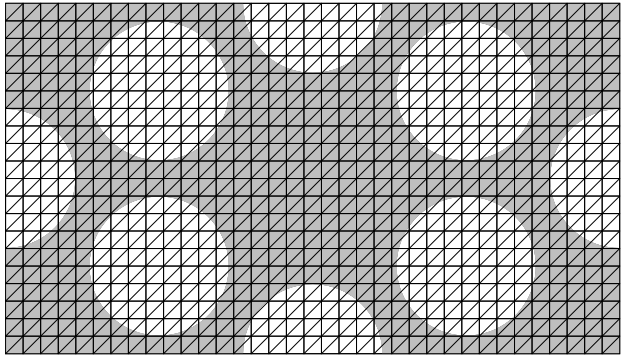

(b) The hexagonal cell

Fig. 2. The regular mesh of the configuration 
of the configuration is illustrated in Fig. 2. The level-set function for elliptic inclusions is defined by

$$
\phi(\mathbf{x})=\min _{k=1, \ldots, n_{c}}\left(\sqrt{\left(x-x_{c k}\right)^{2}+\left(y-y_{c k}\right)^{2}}-r_{k}\right),
$$

where $\left(x_{c k}, y_{c k}\right)$ being the centers of inclusions, $r_{k}$ are radius of circular (spherical) inclusions; $n_{c}$ being number of inclusions. The level set function $\phi(\mathbf{x})$ and the enrichment function $\psi(\mathbf{x})$ defined in Eq. (10) are illustrated in Fig. 3.

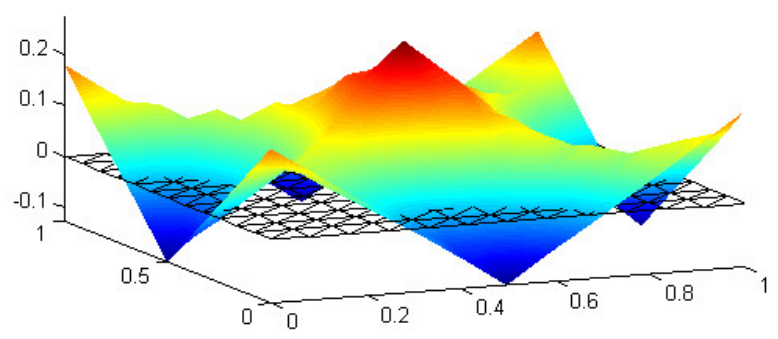

(a)

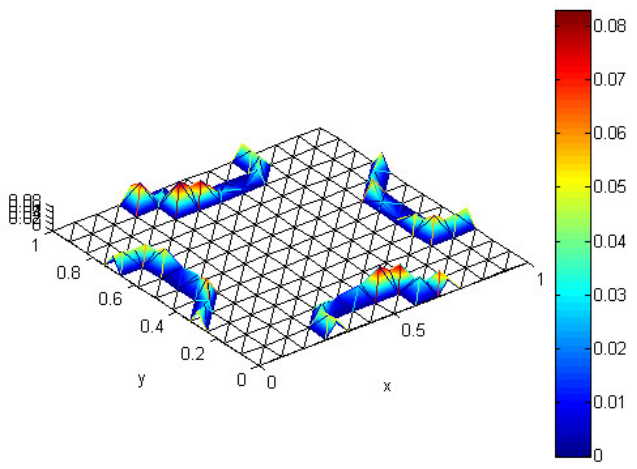

(b)

Fig. 3. (a) Level-Set functions of elliptic inclusions; (b) Enrichment function corresponding to above level-set functions

For numerical calculations, we take the material parameters given in Tab. 1 .

Table 1. Material parameters for numerical calculations

\begin{tabular}{|l|l|l|l|l|}
\hline (a) & $c_{M}=7$ & $c_{I 1}=1$ & $c_{I 2}=21$ & $\overline{c_{I}}=5.44$ \\
\hline (b) & $c_{M}=1$ & $c_{I 1}=7$ & $c_{I 2}=21$ & $\overline{c_{I}}=10.73$ \\
\hline (c) & $c_{M}=21$ & $c_{I 1}=1$ & $c_{I 2}=7$ & $\overline{c_{I}}=3.64$ \\
\hline
\end{tabular}

The equivalent circular (spherical) inclusion has the conductivity $\bar{c}_{I}$ according to (3). The obtained effective conductivity is shown in the Fig. 4, FEM and Equiv, corresponding to the finite element results for original and equivalent configuration, are compared with Maxwell approximation using equivalent inclusion (MA), Hashin-Shtrikman bounds (HSU-Upper bound; HSL-Lower bound). The numerical results lie between the bounds. Both Equiv and MA are close to exact FEM, but MA is less close, as expected (because MAE is a double approximation). The approximations become less accurate at higher volume fractions of the inclusion phases, as expected, because of interactions between the inclusions that no simple approximations can cover. Still, the approximation appears relatively at small to intermediate values of volume proportions of the inclusions. For the hexagonal microstructure, we also take the material parameters given in the table, and 


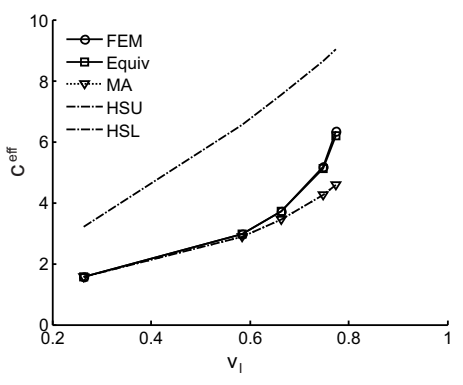

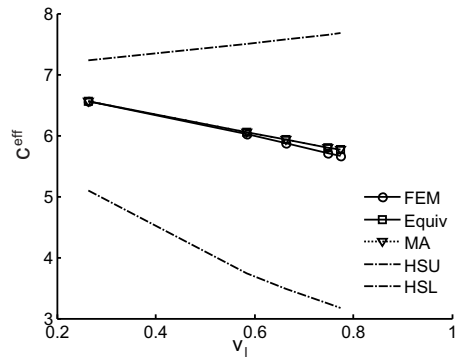

b)

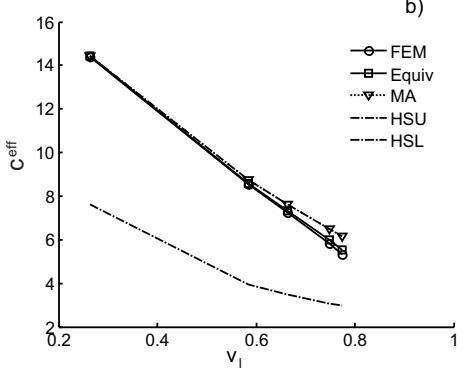

c)

Fig. 4. The graphics of the effective conductivity for square cell. (a) $C_{M}=1 ; C_{I 1}=7 ; C_{I 2}=21$;

(b) $C_{M}=7 ; C_{I 1}=1 ; C_{I 2}=21 ;$ (c) $C_{M}=21 ; C_{I 1}=1 ; C_{I 2}=7$
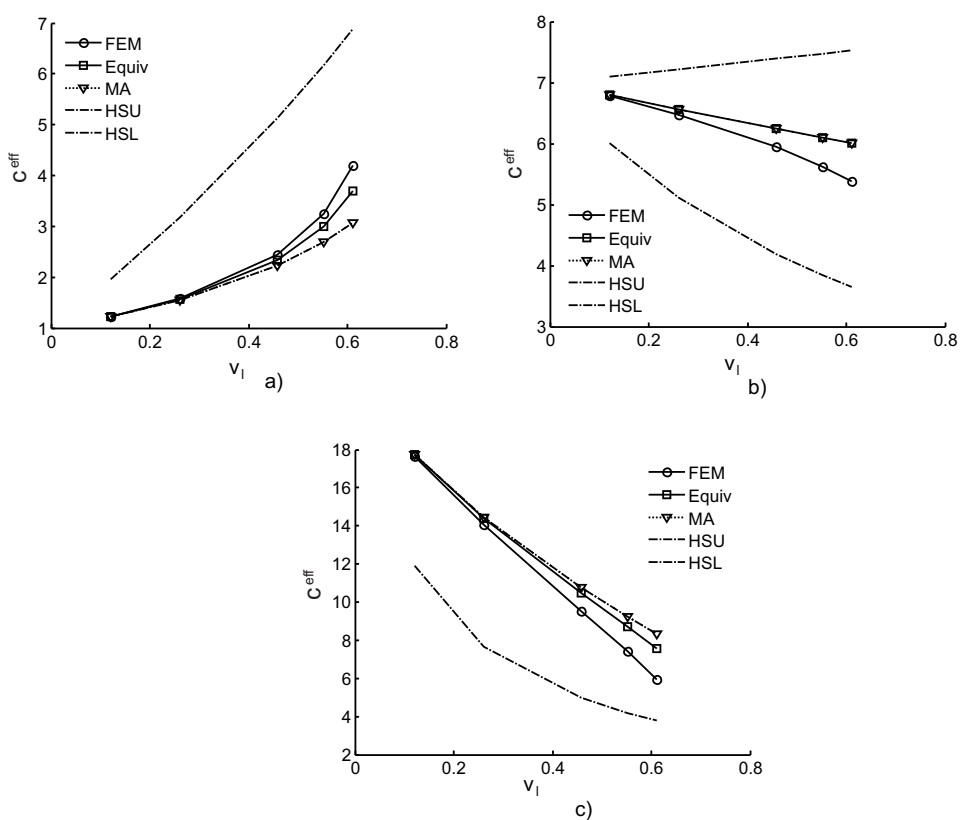

Fig. 5. The graphics of the effective conductivity for hexagonal cell. (a) $C_{M}=1 ; C_{I 1}=7$; $C_{I 2}=21 ;(\mathrm{b}) C_{M}=7 ; C_{I 1}=1 ; C_{I 2}=21 ;$ (c) $C_{M}=21 ; C_{I 1}=1 ; C_{I 2}=7$ 


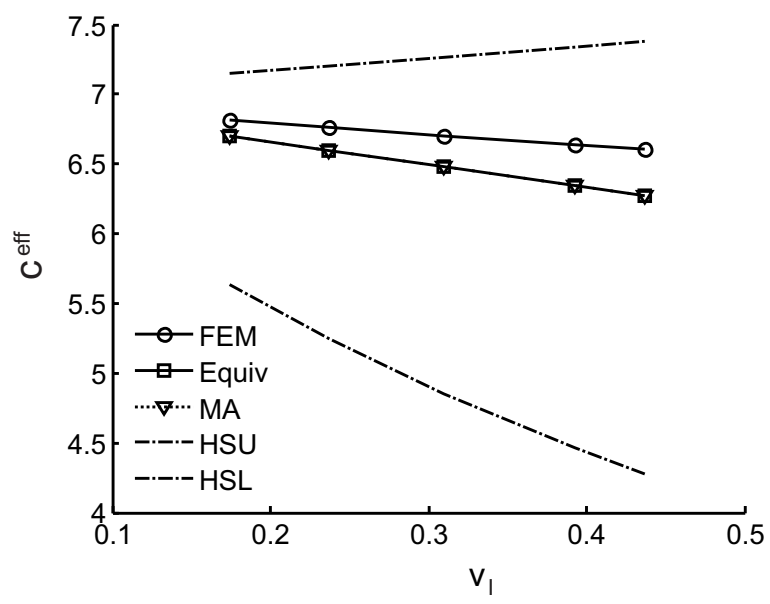

Fig. 6. Graphics of the effective conductivity with random cell. $C_{M}=7 ; C_{I 1}=1 ; C_{I 2}=21$

get the results presented in Fig. 5. The differences seem to be larger because of agglomeration of the inclusion particles. For the random model with high complexity, the results presented in the Fig. 6 indicate that the numerical equivalent-inclusions result (Equiv) and Maxwell approximation (MA) almost coincide, and both are close to FEM results for original composites.

\section{CONCLUSION}

The main idea here is to replace the original composite with disorderly-orientedanisotropic circular (or spherical) inclusion by the equivalent isotropic circular (or spherical) inclusions composite, to which much more simple approximations can be applied to estimate the effective conductivity. The proposed approach is based on equalizing the dilute solution results for the original anisotropic inclusions and that for the circular isotropic inclusions of the same volume fractions, but with some equivalent conductivity. It permits to substitute the original composite by an equivalent composite containing only circular (or spherical) inclusions. Then available effective medium schemas for circular (or spherical) inclusion composites, such as the Maxwell one can be applied to the equivalent medium. The numerical example have demonstrated the robustness, efficiency and accuracy of the approach. Clearly the approach can be extended also for macroscopically isotropic composites with anisotropic inclusions of non-circular (nonspherical) forms.

\section{ACKNOWLEDGEMENT}

This research is funded by Vietnam National Foundation for Science and Technology Development (NAFOSTED) under grant number 107.02-2014.08 


\section{REFERENCES}

[1] D. C. Pham. Bounds for the effective conductivity and elastic moduli of fully-disordered multicomponent materials. Archive for Rational Mechanics and Analysis, 127, (2), (1994), pp. 191198. doi:10.1007/bf00377661.

[2] D. C. Pham. Bounds for the effective properties of isotropic composite and poly-crystals. PhD thesis, Hanoi, (1996).

[3] N. Phan-Thien and D. C. Pham. Differential multiphase models for polydispersed spheroidal inclusions: thermal conductivity and effective viscosity. International Journal of Engineering Science, 38, (1), (2000), pp. 73-88. doi:10.1016/s0020-7225(99)00016-6.

[4] C. F. Dunant, B. Bary, A. B. Giorla, C. Péniguel, J. Sanahuja, C. Toulemonde, A.-B. Tran, F. Willot, and J. Yvonnet. A critical comparison of several numerical methods for computing effective properties of highly heterogeneous materials. Advances in Engineering Software, 58, (2013), pp. 1-12. doi:10.1016/j.advengsoft.2012.12.002.

[5] B. Bary, M. B. Haha, E. Adam, and P. Montarnal. Numerical and analytical effective elastic properties of degraded cement pastes. Cement and Concrete Research, 39, (10), (2009), pp. 902912. doi:10.1016/j.cemconres.2009.06.012.

[6] A. B. Tran, J. Yvonnet, Q.-C. He, C. Toulemonde, and J. Sanahuja. A multiple level set approach to prevent numerical artefacts in complex microstructures with nearby inclusions within XFEM. International Journal for Numerical Methods in Engineering, 85, (11), (2011), pp. 1436-1459. doi:10.1002/nme.3025.

[7] H. Moulinec and P. Suquet. A fast numerical method for computing the linear and nonlinear mechanical properties of composites. Comptes Rendus De L'académie Des Sciences. Série Ii, Mécanique, Physique, Chimie, Astronomie, 318, (11), (1994), pp. 1417-1423.

[8] J. C. Michel, H. Moulinec, and P. Suquet. A computational scheme for linear and non-linear composites with arbitrary phase contrast. International Journal for Numerical Methods in Engineering, 52, (1-2), (2001), pp. 139-160. doi:10.1002/nme.275.

[9] J. Sanahuja and C. Toulemonde. Numerical homogenization of concrete microstructures without explicit meshes. Cement and Concrete Research, 41, (12), (2011), pp. 1320-1329. doi:10.1016/j.cemconres.2011.03.023.

[10] A. N. Norris, A. J. Callegari, and P. Sheng. A generalized differential effective medium theory. Journal of the Mechanics and Physics of Solids, 33, (6), (1985), pp. 525-543. doi:10.1016/00225096(85)90001-8.

[11] M. Milgrom and S. Shtrikman. A layered-shell model of isotropic composites and exact expressions for the effective properties. Journal of Applied Physics, 66, (8), (1989), pp. 3429-3436. doi:10.1063/1.344097.

[12] D. C. Pham. Estimations for the overall properties of some locally-ordered composites. Acta Mechanica, 121, (1-4), (1997), pp. 177-190. doi:10.1007/bf01262531.

[13] D. C. Pham and S. Torquato. Strong-contrast expansions and approximations for the effective conductivity of isotropic multiphase composites. Journal of Applied Physics, 94, (10), (2003), pp. 6591-6602. doi:10.1063/1.1619573.

[14] D. C. Pham. Weighted effective medium approximations for conductivity of random composites. International Journal of Heat and Mass Transfer, 51, (13), (2008), pp. 3355-3361. doi:10.1016/j.ijheatmasstransfer.2007.11.035.

[15] H. Le Quang, G. Bonnet, and D. C. Pham. Bounds and correlation approximation for the effective conductivity of heterogeneous plates. Physical Review E, 84, (6), (2011), p. 061153. doi:10.1103/physreve.84.061153. 
[16] D. C. Pham, A. B. Tran, and Q. H. Do. On the effective medium approximations for the properties of isotropic multicomponent matrix-based composites. International Journal of Engineering Science, 68, (2013), pp. 75-85. doi:10.1016/j.ijengsci.2013.03.007.

[17] D. C. Pham. Essential solid mechanics. Institute of Mechanics, Hanoi, (2013).

[18] H. Le Quang, D. C. Pham, G. Bonnet, and Q.-C. He. Estimations of the effective conductivity of anisotropic multiphase composites with imperfect interfaces. International Journal of Heat and Mass Transfer, 58, (1), (2013), pp. 175-187. doi:10.1016/j.ijheatmasstransfer.2012.11.028.

[19] S. Torquato. Random heterogeneous media. New York, Springer, (2013).

[20] N. Sukumar, D. L. Chopp, N. Moës, and T. Belytschko. Modeling holes and inclusions by level sets in the extended finite-element method. Computer Methods in Applied Mechanics and Engineering, 190, (46), (2001), pp. 6183-6200. doi:10.1016/s0045-7825(01)00215-8.

[21] J. Yvonnet, Q. C. He, and C. Toulemonde. Numerical modelling of the effective conductivities of composites with arbitrarily shaped inclusions and highly conducting interface. Composites Science and Technology, 68, (13), (2008), pp. 2818-2825. doi:10.1016/j.compscitech.2008.06.008.

[22] A. B. Tran, J. Yvonnet, Q.-C. He, C. Toulemonde, and J. Sanahuja. A multiple level set approach to prevent numerical artefacts in complex microstructures with nearby inclusions within XFEM. International Journal for Numerical Methods in Engineering, 85, (11), (2011), pp. 1436-1459. doi:10.1002/nme.3025.

[23] S. Mohammadi. Extended finite element method. Blackwell Publishing Ltd, (2008). doi:10.1002/9780470697795.

[24] J. Yvonnet, H. L. Quang, and Q.-C. He. An XFEM/level set approach to modelling surface/interface effects and to computing the size-dependent effective properties of nanocomposites. Computational Mechanics, 42, (1), (2008), pp. 119-131. doi:10.1007/s00466-008-0241-y. 